\title{
Rheological Percolation of Cellulose Nanocrystals in Biodegradable Poly(butylene succinate) Nanocomposites: A Novel Approach for Tailoring the Mechanical and Hydrolytic Properties
}

\author{
Hyo Jeong Kim ${ }^{1}$ \\ Yun Hyeong Choi ${ }^{1}$ \\ ji Hun Jeong ${ }^{1}$ \\ ${\text { Hyeri } \mathrm{Kim}^{2}}^{2}$ \\ Ho Sung Yang ${ }^{2}$ \\ Sung Yeon Hwang ${ }^{2,3}$ \\ Jun Mo $\mathrm{Koo}^{*, 2}$ \\ Youngho Eom*,1
}

\author{
${ }^{1}$ Department of Polymer Engineering, Pukyong National University, Busan 48513, Korea \\ ${ }^{2}$ Research Center for Bio-based Chemistry, Korea Research Institute of Chemical Technology \\ (KRICT), Ulsan 44429, Korea \\ Advanced Materials and Chemical Engineering, University of Science and Technology (UST), \\ Daejeon 34113, Korea
}

Received April 13, 2021 / Revised July 13, 2021 / Accepted August 12, 2021

\begin{abstract}
Although biodegradable plastics are gradually emerging as an effective solution to alleviate the burgeoning plastic pollution, their performance is currently trivial for commercialization. A proposed two-pronged strategy to overcome this limitation includes (1) preparation of the nanocomposites from biorenewable nanofillers to preserve their biodegradability and (2) tailoring their properties to meet the diverse demands in various applications. Herein, we report the preparation of biodegradable nanocomposites composed of poly(butylene succinate) (PBS) and cellulose nanocrystals (CNCs) (loading of 0.2-3.0 wt\%) and propose a rheological strategy to tailor their performances. Depending on the shear frequencies, the rheological evaluation revealed two percolation thresholds at approximately 0.8 and $1.5 \mathrm{wt} \%$. At high shear frequencies, the disappearance of the first threshold ( $0.8 \mathrm{wt} \%$ ) and the sole persistence of the second one $(1.5 \mathrm{wt} \%)$ indicated the collapse of the immature network of partially interconnected CNCs. The tensile and hydrolytic properties of the nanocomposites were found to undergo drastic changes at the thresholds. The tensile strength increased by $17 \%$ (from 33.3 to $39.2 \mathrm{MPa}$ ) up to 0.8 $w t \%$ CNC loading. However, the reinforcing efficiency of CNC decreases sharply

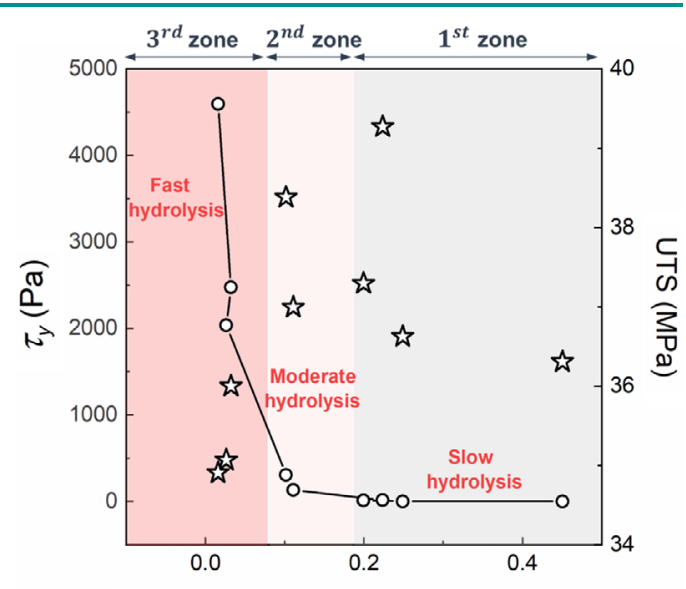

Efficiency for UTS ([fold-1].wt.-1 ${ }^{-1}$ with further incorporation, reaching nearly zero at $1.5 \mathrm{wt} \%$. On the other hand, hydrolytic degradation of the nanocomposites was rapidly accelerated above $1.5 \mathrm{wt} \%$ CNC loading. Therefore, a thorough understanding of the rheological properties of nanocomposites is essential for the design and development of materials with tailored properties.
\end{abstract}

Keywords: poly(butylene succinate), PBS-CNC nanocomposite, cellulose nanocrystals, rheological percolation threshold.

\section{Introduction}

Petroleum-derived plastics have become an indispensable commodity in our daily lives owing to their lighter weight, easier processing, lower production cost, and higher versatility when compared to metals and ceramics. ${ }^{1,2}$ Accordingly, the cumulative environmental damage caused by non-degradable plastics is largely ignored because they offer such high convenience of use. Plastic pollution of soil, atmosphere, and marine systems has been rising continuously, being further aggravated by the recent coronavirus pandemic (COVID-19).,3 Biodegradable plastics are increasingly considered as an effective remedy for the malaise of plastic pollution, especially to address the challenge posed by the rapid growth

Acknowledgments: This research was supported by the Pukyong National University Research Fund in 2019 (C-D-2019-1540) and the National Research Foundation of Korea (NRF-2020R1C1C1009340).

*Corresponding Authors: Jun Mo Koo (jmkoo@krict.re.kr),

Youngho Eom (eomyh@pknu.ac.kr) of disposable products. ${ }^{5,6}$ In the 1990s, popular biodegradable polymers, including poly(lactic acid), polycaprolactone, poly(butylene succinate) (PBS), and poly(butylene adipate-co-terephthalate), were commercialized as alternatives to the non-biodegradable petroleum-based plastics. However, inferior mechanical performance and poor durability limited their large-scale industrial applicability despite being promising materials for sustainability. $^{7}$

Preparing nanocomposites by incorporating reinforcing fillers offers a rational solution to overcome certain performance limitations. Polymer nanocomposites are generally composed of a polymer matrix and at least one nano-sized filler. Numerous studies have been dedicated to nanocomposites of biodegradable polymers employing various types of nano-fillers, such as clays,${ }^{8-11}$ carbon nanotubes, ${ }^{12}$ graphene (or graphene oxide), glass fiber, ${ }^{13}$ and metal nanoparticles. ${ }^{14}$ Nonetheless, the use of such inorganic fillers raises similar environmental concerns associated with conventional plastics, as they are neither degradable nor pyrolizable. ${ }^{15}$ Moreover, the fine particulate matter generated 
upon incineration of inorganic filler-reinforced composites gets suspended in the air, becoming a serious cause of lung diseases. ${ }^{16}$ In other words, the non-degradable nature of inorganic fillers renders the sustainability of biodegradable polymers useless.

Recently, the incorporation of biorenewable nanofibers into biodegradable polymers has emerged as a promising sustainable alternative to inorganic fillers. ${ }^{17-19}$ Typical examples of natural nanofibers include cellulose nanocrystals (CNCs), cellulose nanofibers, and chitin or chitosan nanowhiskers. ${ }^{20-22}$ Among them, CNCs have gained significant attention as nano-fillers owing to their high aspect ratio, high crystallinity (54-88\%), and the presence of numerous polar functional groups that facilitate secondary interactions with the organic polymer matrix ${ }^{23,24}$ We have previously reported the in situ preparation of PBS nanocomposites using CNCs. ${ }^{6,725}$ Poor tear resistance renders neat PBS unsuitable for manufacturing disposable plastic bags. ${ }^{6,26}$ However, the introduction of CNCs significantly improves the mechanical strength under both tensile and tearing conditions. ${ }^{6}$ In this study, PBS-CNC nanocomposites were fabricated via solution blending methods. The CNC loading was varied from $0-3.0 \mathrm{wt} \%$ of the total polymer content. In addition, we propose a rheological strategy to tailor the mechanical and hydrolytic properties of PBS-CNC nanocomposites. The results of this study revealed that the rheological percolation behavior of CNCs has a profound effect on the reinforcing efficiency.

\section{Experimental}

\subsection{Materials}

Commercial-grade PBS (MI4) was purchased from Ankor Bioplastics (Korea). The spray-dried CNC powder was purchased from CelluForce Co. (Canada). EP grade dimethyl sulfoxide (DMSO, >99\%) was purchased from Duksan Co. (Korea). Sodium hydroxide $(\mathrm{NaOH})$ was purchased Sigma Aldrich (USA). All the chemicals were used without further purification.

\subsection{Preparation of PBS-CNC nanocomposites}

Owing to the infusible character of CNCs, the solution blending method was adopted to fabricate the PBS-CNC nanocomposites. Initially, CNC was pre-dispersed in DMSO as a co-solvent at a specific concentration using bath-type sonication until a transparent solution appeared, and then PBS was dissolved in CNC/ DMSO solution at $90{ }^{\circ} \mathrm{C}$ for $2 \mathrm{~h}$. The CNC loading relative to the total polymer content was varied from $0-3.0 \mathrm{wt} \%$. PBS solutions with various CNC concentrations were poured into Deionized (DI) water under mechanical stirring, and the precipitate was separated. The PBS-CNC powders thus obtained were filtered and washed with DI water and ethanol several times. Finally, the powders were dried in a vacuum oven at $60{ }^{\circ} \mathrm{C}$ for $24 \mathrm{~h}$ to remove residual solvent or water. The PBS-CNC nanocomposite films intended for mechanical testing were fabricated by hotpressing the PBS-CNC powder in a Teflon sheet-mold at $180{ }^{\circ} \mathrm{C}$ for $10 \mathrm{~min}$, followed by quenching in cold water for $5 \mathrm{~min}$. The prepared film size was $8 \mathrm{~cm} \times 8 \mathrm{~cm}$.

\subsection{Characterization}

The mechanical properties of the PBS-CNC nanocomposite films were measured using a universal testing machine (Instron 5943, USA) loaded with a $1 \mathrm{kN}$ load cell. According to the ISO 37-4 guideline, 1-mm-thick dumbbell-type specimens were cut. The crosshead speed and gauge length were $100 \mathrm{~mm} \mathrm{~min}^{-1}$ and $12 \mathrm{~mm}$, respectively. All specimens were conditioned at room temperature $\left(25^{\circ} \mathrm{C}\right)$ and $35 \%$ relative humidity.

Wide-angle X-ray scattering (WAXS) measurements were conducted using synchrotron radiation $(\lambda=1.28 \AA)$ on the $3 \mathrm{C}$ SAXS I beamline of the Pohang Accelerator Laboratory. The wavelength and pixel size were $1.229 \AA$ and $0.079 \mathrm{~mm}$, respectively. The sample-to-detector distance was $0.012 \mathrm{~m}$ for the WAXS measurements.

The rheological properties of the PBS-CNC series were evaluated in the molten state using a rotational rheometer (MCR 302 Anton Paar, Austria). A parallel plate geometry was used for the rheological measurements with a gap and strain of $1.0 \mathrm{~mm}$ and $5 \%$, respectively. The oscillation frequency sweeps were performed at $160{ }^{\circ} \mathrm{C}$ in the angular frequency range of 0.05-500 $\mathrm{rad} \mathrm{s}^{-1}$. Prior to measurement, the melt was placed between the plates for $5 \mathrm{~min}$ to completely relax the residual stresses.

The chemical hydrolysis of the nanocomposite films was conducted in an aqueous $0.1 \mathrm{~N} \mathrm{NaOH}$ solution. Square-shaped sample specimens of side $3 \mathrm{~cm}$ and thickness $0.11 \pm 0.04 \mathrm{~mm}$ were cut from the films. The specimen was placed in a vial and immersed for $6 \mathrm{~d}$. After a predetermined time, the chemically degraded PBS-CNC nanocomposite films were removed, washed with DI water, and then dried using a heat gun to remove surface water. The weight loss was evaluated by the following equation;

$W_{\text {loss }}(\%)=\frac{W_{0}-W_{1}}{W_{0}} \times 100$

where $W_{0}$ and $W_{1}$ are the weights of the specimen before and after degradation, respectively. In order to observe the surface morphologies of chemically degraded specimens, field-emission SEM was performed using a Tescan MIRA3 (Czech Republic) microscope. Prior to the observation, the degraded films were coated with platinum to obtain high quality images.

\section{Results and discussion}

\subsection{Mechanical and hydrolytic properties of PBS-CNC nanocomposites}

Solution blending is the most effective way to achieve a homogeneous dispersion of CNCs while preparing polymer nanocomposites due to the infusibility of cellulosic materials. ${ }^{27}$ DMSO was employed as a co-solvent for the solution mixing of PBS and CNCs. The preparation procedure is schematically depicted in Figure 1(a). A certain amount of CNCs was dispersed in DMSO by sonication, and then PBS was dissolved at $90{ }^{\circ} \mathrm{C}$ for $2 \mathrm{~h}$ under mechanical stirring. The solution thus obtained was poured into DI water, and the resulting precipitate was filtered and vacuum-dried. The CNC loading relative to the total polymer content was varied from 0-3.0 wt\%. The PBS-CNC nanocomposites will henceforth 

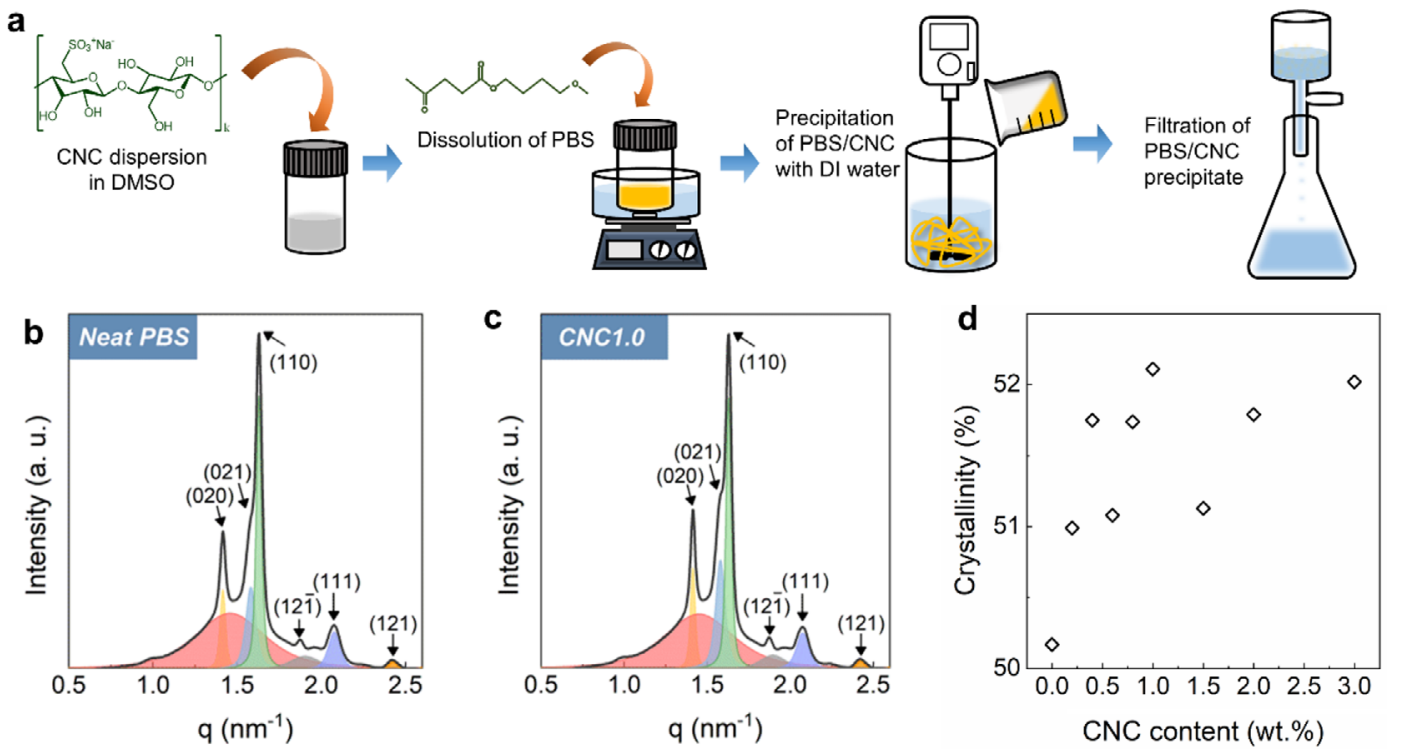

Figure 1. (a) Preparation procedure of PBS-CNC nanocomposites via solution blending method, deconvoluted 1D WAXS patterns of (b) neat PBS and (c) CNC1.0, and (c) the crystallinity of neat PBS and PBS-CNC nanocomposites calculated from the analysis of the X-ray scattering patterns.

be referred to as "CNCn" with $n$ denoting the $\mathrm{CNC}$ loading (in wt\%). The effect of CNC on the crystallinity was investigated by deconvoluting the 1D WAXS patterns of neat PBS and PBSCNC nanocomposites into six crystalline peaks ((020), (021), (110),

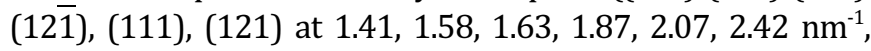
respectively), and an amorphous band (Figures 1(b) and 1(c)). As shown in Figure 1(d), the incorporation of CNC increases the crystallinity by $2 \%$ (52.1\% for CNC1.0 and $50.1 \%$ for neat PBS).

Figures 2(a)-(c) show the dependence of the mechanical properties on the CNC loading of the PBS-CNC nanocomposites. As shown in Figure 2(a), Young's modulus of the PBS-CNC nanocomposites increases monotonically with the CNC loading, an expected trend given the high stiffness of CNC molecules in comparison to the polymer host matrix. ${ }^{28}$ By contrast, the ultimate tensile strength (UTS) varies non-monotonically with CNC loading (Figure 2(b)), exhibiting a 1.17-fold increase until $0.8 \mathrm{wt} \%$ (33.3 MPa for neat PBS to 39.2 MPa for CNC0.8), followed by a gradual decrease upon further incorporation up to $3 \mathrm{wt} \%$ (34.9 MPa for CNC3.0). In terms of toughness (Figure 2(c)), it exhibits a sudden drop above $1.5 \mathrm{wt} \%$ of CNC loading. It can be conjectured that the drastic reduction in the UTS and toughness is closely related to the reduction in the reinforcing efficiency of the CNCs above
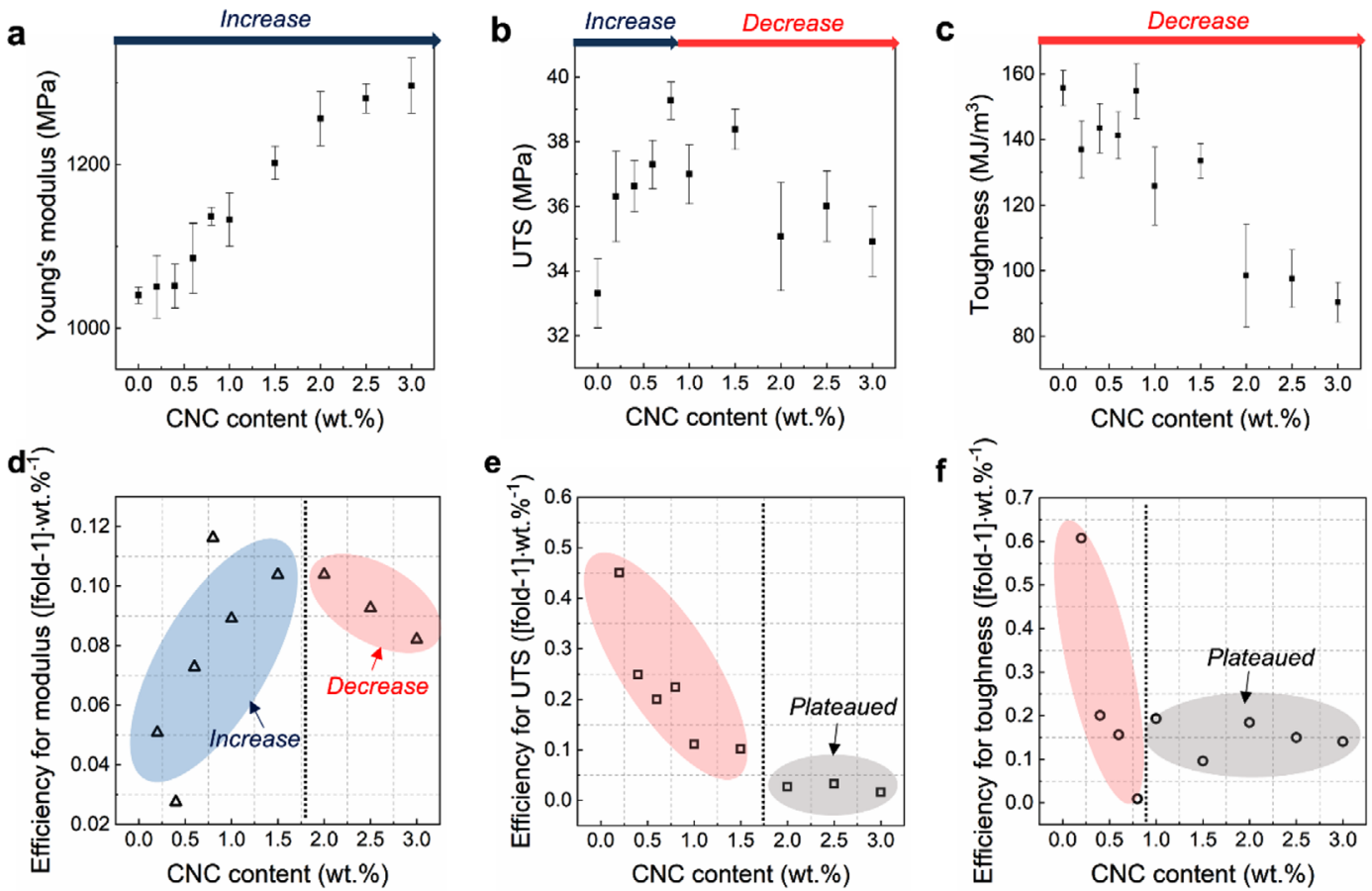

Figure 2. Mechanical properties of neat PBS and PBS-CNC nanocomposites; Plots of (a) Young's modulus, (b) UTS, and (c) toughness of PBS-CNC nanocomposites as a function of the filler content, and dependence of the reinforcing efficiencies of CNCs on the CNC loading with respect to the (d) Young's modulus, (e) UTS, and (f) toughness. 
a certain threshold loading.

To evaluate the reinforcing efficiency of CNCs in PBS, the increase extent of Young's modulus, UTS, and toughness per unit of CNC loading ( $1 \mathrm{wt} \%$ ) [(fold- 1 ) wt $\%^{-1}$ ] was calculated and plotted as a function of the CNC loading (Figures 2(d)-(f)). ${ }^{21}$ Interestingly, we find that the reinforcing efficiency exhibits a marked trend change at a critical CNC loading. With respect to Young's modulus, reinforcing efficiency increases up to $1.5 \mathrm{wt} \% \mathrm{CNC}$ loading, beyond which it starts decreasing. In case of UTS, the reinforcing efficiency reaches almost zero above $1.5 \mathrm{wt} \%$. However, with regard to toughness, the efficiency plateaus above $0.8 \mathrm{wt} \%$. Therefore, we identify two critical CNC loadings at 0.8 and $1.5 \mathrm{wt} \%$, at which the reinforcing efficiency of the CNCs changes significantly, leading to a drastic change in the mechanical performance of PBS-CNC nanocomposites.

The hydrolysis of PBS-CNC nanocomposites was conducted

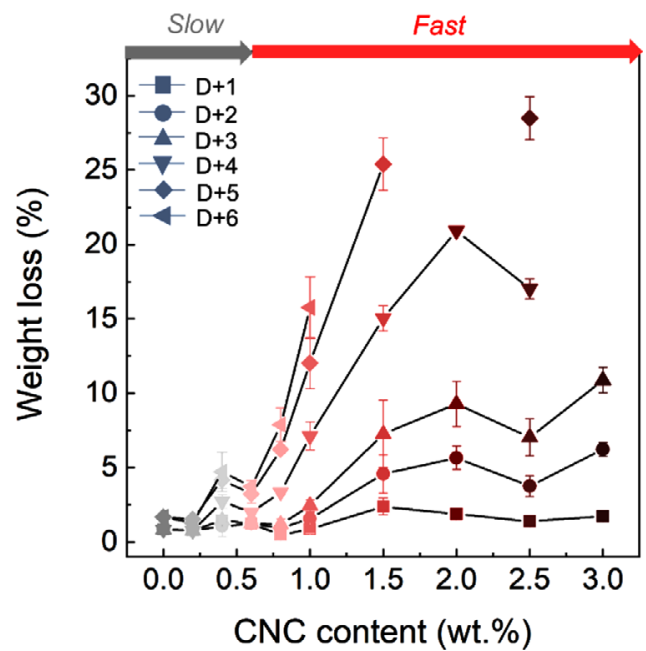

Figure 3. Weight loss of neat PBS and PBS-CNC nanocomposite films upon chemical hydrolysis. in a chemical medium consisting of $\mathrm{NaOH}(0.1 \mathrm{~N}){ }^{29,30}$ As shown in Figure 3, the neat PBS and PBS-CNC nanocomposite specimens undergo weight loss of less than $5 \%$ (0.118 to $0.117 \mathrm{~g}$ for the neat PBS and 0.132 to $0.129 \mathrm{~g}$ for $\mathrm{CNC} 0.6$ ) upon chemical degradation for 6 days in the CNC loading from 0.0-0.6 wt $\%$, whereas the weight loss of the nanocomposites becomes significant above $0.8 \mathrm{wt} \%$. The weight loss for CNC1.0 and CNC1.5 was about $15 \%$ ( 0.149 to $0.127 \mathrm{~g}$ after 6 days) and $25 \%$ (0.148 to $0.112 \mathrm{~g}$ after 5 days), respectively. This suggests that the chemical degradation of PBS-CNC nanocomposites rapidly accelerates above the critical $\mathrm{CNC}$ loading of $0.8 \mathrm{wt} \%$. The accelerated degradation of the nanocomposites can be further proven by observing the surface morphologies of the degraded films (Figure 4). Compared to the less-degraded neat PBS and PBS-CNC nanocomposite films even after the hydrolysis for 6 days (below $0.8 \mathrm{wt} \%$ ), the films above the critical CNC content (CNC1.0, 2.0, and 3.0) exhibits peculiar morphology because of an intensive chemical etching by hydroxyl anions. This cracked morphology is well matched with the substantial weight loss upon the hydrolysis.

\subsection{Rheological percolation analysis of PBS-CNC nano- composites}

It is reasonable to expect that the significant change in the reinforcing efficiency of the CNCs at a certain loading could be correlated with their percolation behavior in the polymer matrix. Figures 5(a) and 5(b) show the dynamic viscosity $\left(\eta^{\prime}\right)$ and storage modulus $(G)$ curves of neat PBS and PBS-CNC nanocomposites in the molten state at $160{ }^{\circ} \mathrm{C}$. In Figure 5(a), we observe that neat PBS exhibits a Newtonian behavior with the existence of a lower Newtonian flow region (LNFR). However, as the CNC loading increases, the flow behavior changes into the non-Newtonian Bingham body type, wherein the LNFR disappears, and the vis-
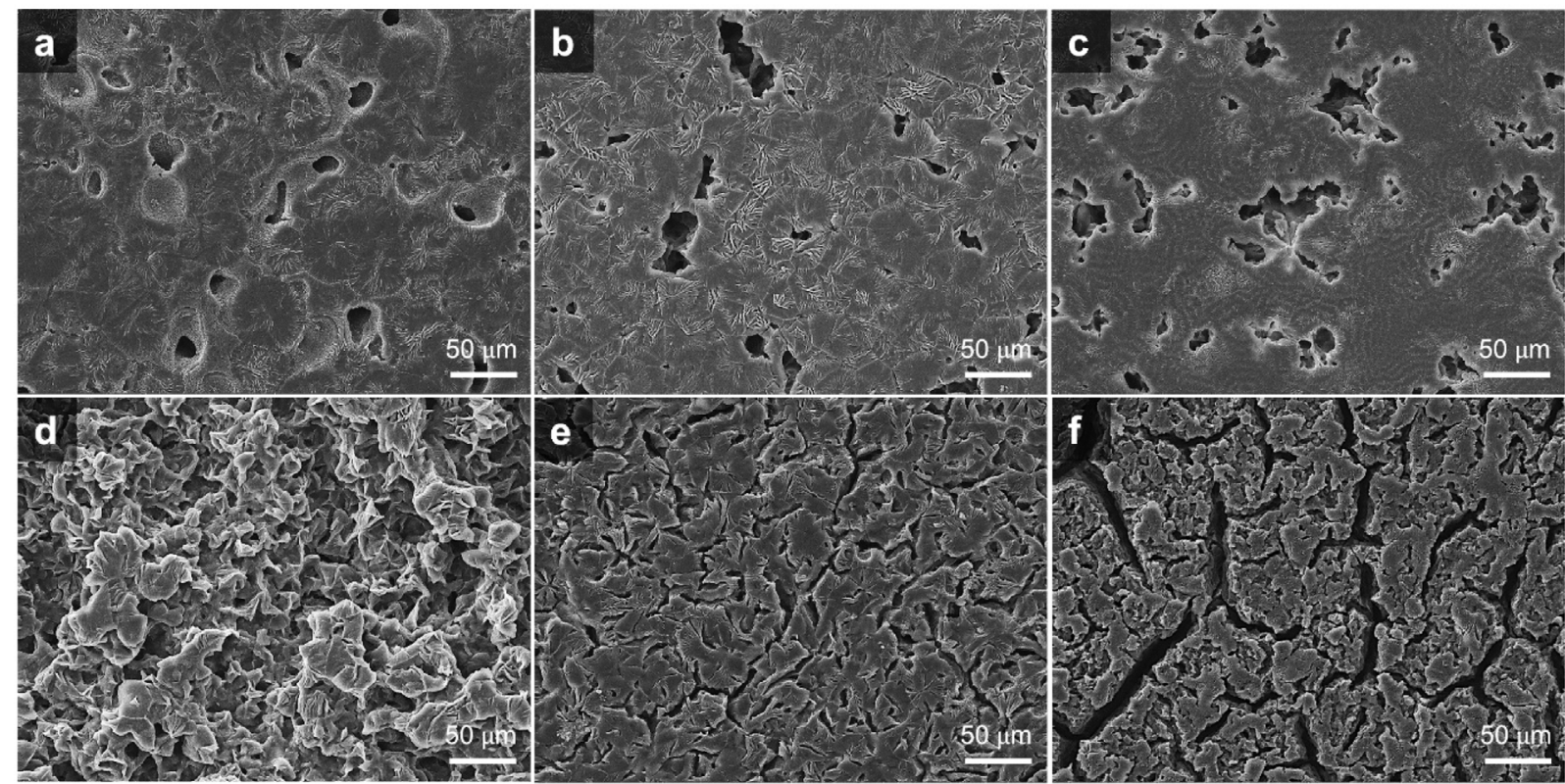

Figure 4. SEM images of degraded films upon chemical hydrolysis; (a) neat PBS, (b) CNC0.4, (c) CNC0.8, (d) CNC1.0, (e) CNC2.0, and (f) CNC3.0. The neat PBS and CNC0.4-1.0 specimens were hydrolyzed for 6 days, whereas CNC2.0 and CNC3.0 were treated for 4 and 3 days, respectively, due to the specimen disintegration. 

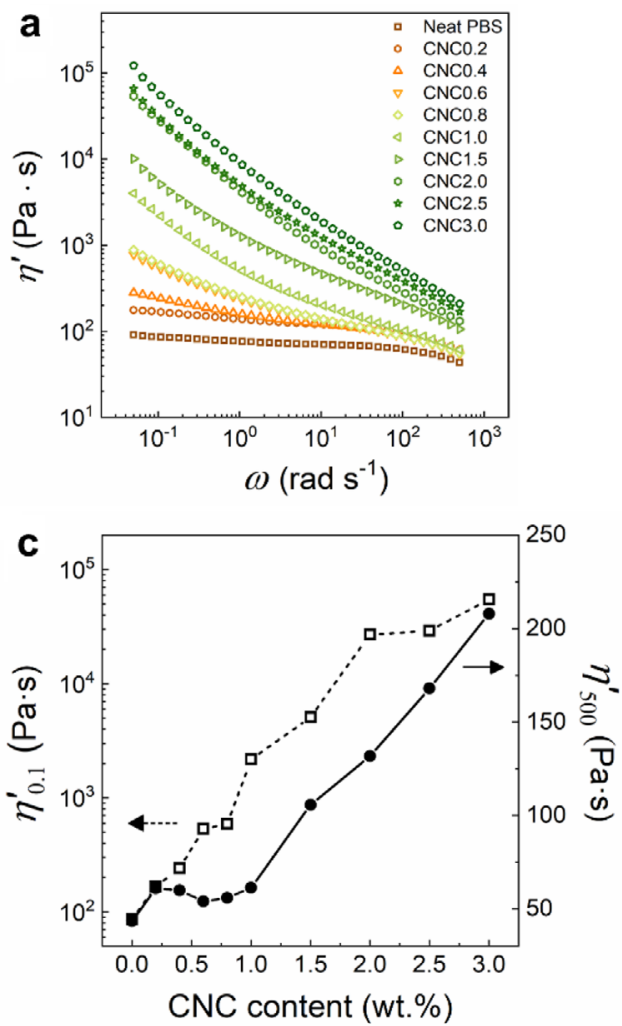
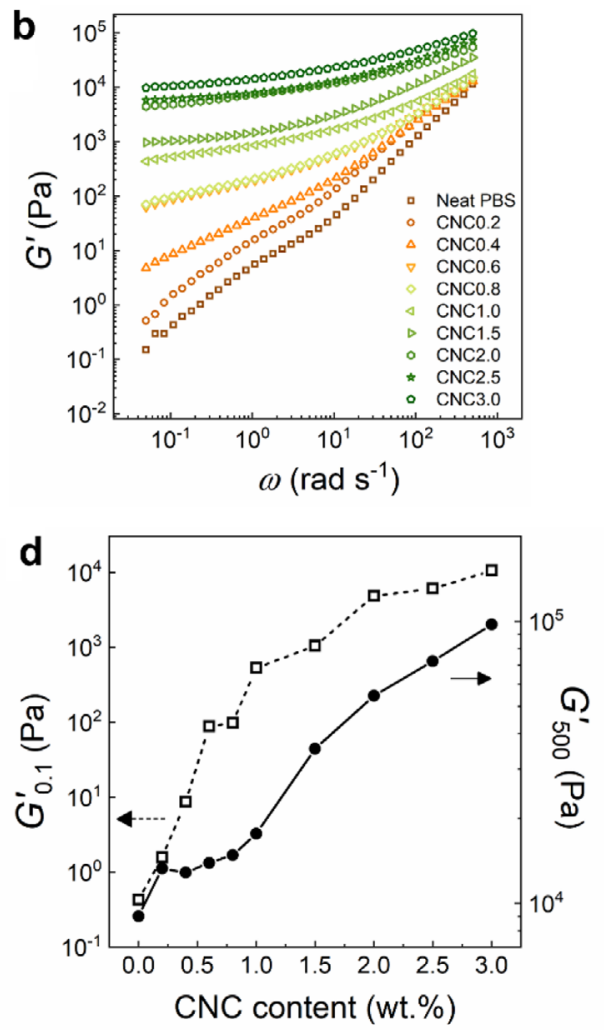

Figure 5. (a) Dynamic viscosity $\left(\eta^{\prime}\right)$ and (b) storage modulus $\left(G^{\prime}\right)$ curves of neat PBS and PBS-CNC nanocomposites at $160{ }^{\circ} \mathrm{C}$. Dependence of (c) $\eta_{0.1}^{\prime}$ and $\eta_{500}^{\prime}\left(\eta^{\prime}\right.$ at 0.1 and $500 \mathrm{rad} \mathrm{s}^{-1}$, respectively), and (d) $G_{0.1}^{\prime}$ and $G_{500}^{\prime}\left(G^{\prime}\right.$ at 0.1 and $500 \mathrm{rad} \mathrm{s}^{-1}$, respectively) on the CNC loading.

cosity shows a sharp decrease even at low frequencies. ${ }^{31,32}$ In addition, $\eta^{\prime}$ increases noticeably over the entire frequency range of observation. Similarly, incorporation of CNC results in a sharp increase in $G^{\prime}$ (Figure 5(b)). To identify the rheological percolation threshold, the $\eta^{\prime}$ and $G^{\prime}$ values at low $\left(0.1 \mathrm{rad} \mathrm{s}^{-1}\right)$ and high $\left(500 \mathrm{rad} \mathrm{s}^{-1}\right)$ frequencies are plotted against the CNC loading in Figures 5(c) and 5(d), respectively. Although both $\eta^{\prime}$ and $G^{\prime}$ exhibit a significant change in their behavior at the vicinity of $1.0 \mathrm{wt} \%$ CNC loading, it still provides insufficient evidence to determine the percolation threshold.

The rheological percolation threshold can be rationalized by analyzing the phase characteristics and homogeneity. Figure 6(a) displays the loss tangent $(\tan \delta$ ) curves of the neat PBS and PBS-CNC nanocomposites. In principle, an increase/decrease in $\tan \delta$ indicates an enhancement in the viscous/elastic response of the material. ${ }^{33,34}$ The $\tan \delta$ values obtained at low $\left(0.08 \mathrm{rad} \mathrm{s}^{-1}\right)$ and high (108 rad s${ }^{-1}$ ) frequencies are plotted against the CNC loading in Figure 6(b). Interestingly, the critical CNC loading was found to be frequency-dependent. At low frequencies, $\tan \delta$ undergoes a sharp reduction until $0.6 \mathrm{wt} \%$, followed by an insignificant change below unity. This suggests that the percolation network that amplifies the phase rigidity is developed around $0.6 \mathrm{wt} \% \mathrm{CNC}$ loading when the fillers are barely oriented in the static state. It is interesting to note that the rheological percolation threshold ( $\sim 0.6 \mathrm{wt} \%$ of CNC) observed in the static state coincides with the critical CNCloading of $\sim 0.8 \mathrm{wt} \%$ in the chemical degradation of the nanocomposites. This reveals that the development of the inter-connected CNC network through the PBS matrix not
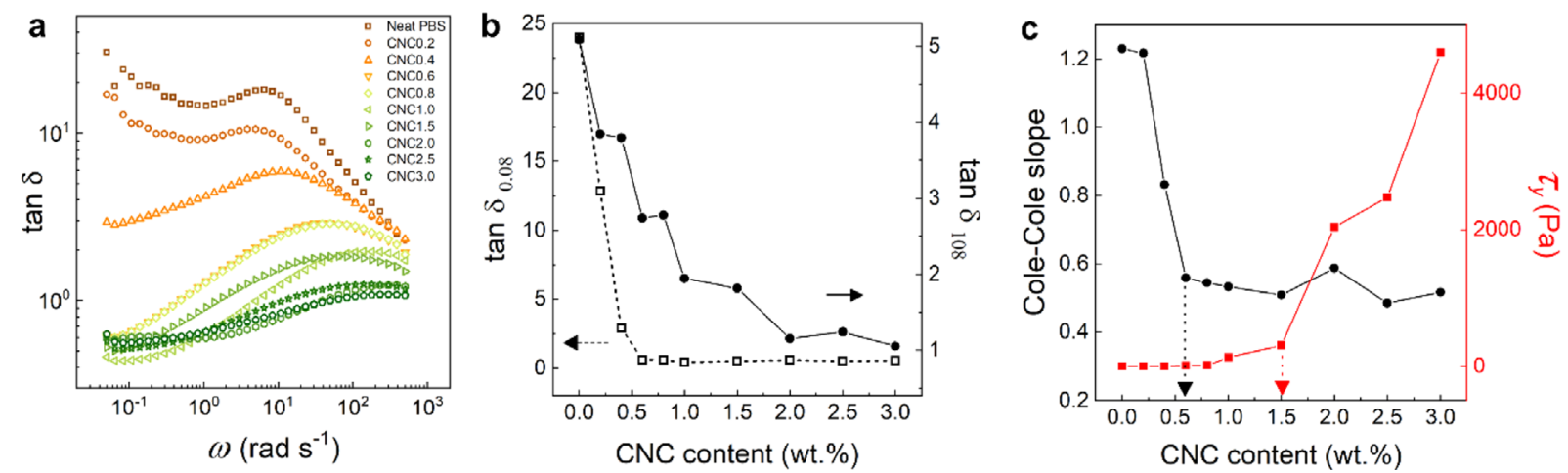

Figure 6. (a) Loss tangent $\left(\tan \delta\right.$ ) curves of neat PBS and PBS-CNC nanocomposites at $160{ }^{\circ} \mathrm{C}$. (b) Dependence of $\tan \delta_{0.08}$ and $\tan \delta_{108}(\tan \delta$ at 0.08 and $108 \mathrm{rad} \mathrm{s}^{-1}$, respectively) on the CNC loading, and (c) slope of the Cole-Cole plots and yield stress ( $\tau_{y}$ ) of neat PBS and PBS-CNC nanocomposites, respectively. 


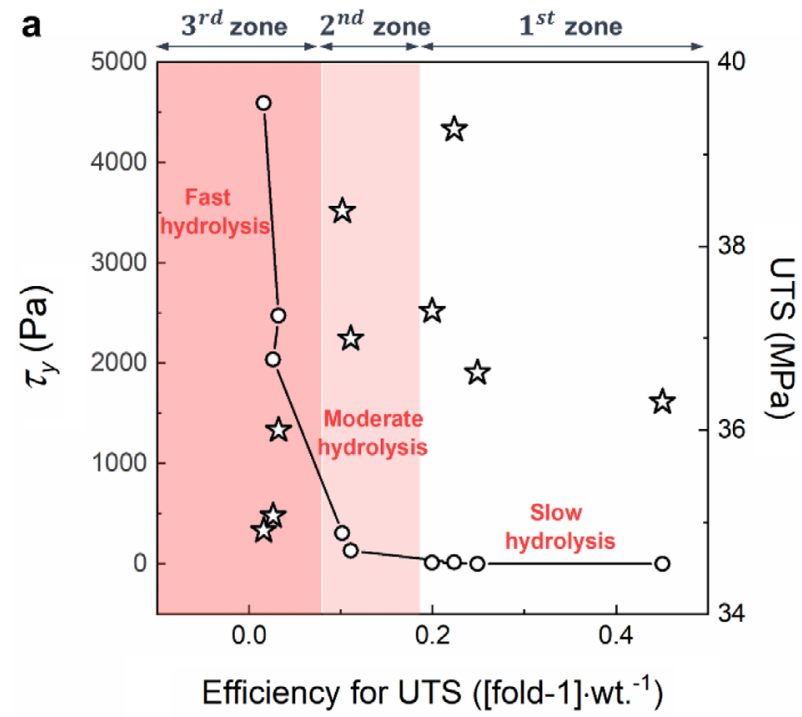

b

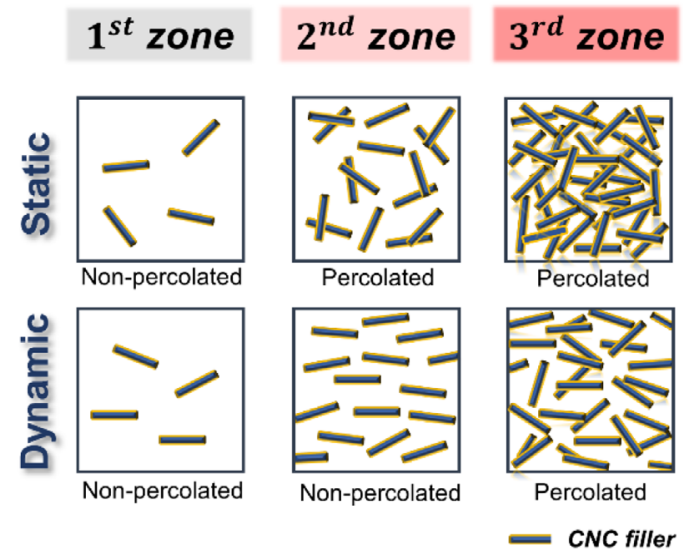

Figure 7. (a) Phase diagram showing the correlation between mechanical performance, chemical hydrolysis and rheological behavior of PBSCNC nanocomposites and (b) percolation networks of CNCs in the PBS matrix are depicted with respect to zones 1-3.

only enhances the elastic properties in terms of rheology but also accelerates the degradation in terms of hydrolysis. As the anisotropic CNC particles are well oriented at high frequencies, $\tan \delta$ decreases gradually until $2.0 \mathrm{wt} \% \mathrm{CNC}$ loading and saturates beyond that. This indicates that the percolation threshold of CNCs shifts to a high loading under dynamic conditions that may be attributed to the effect of filler orientation on the efficiency of network formation. Such percolation networks are known to arise from the interconnection of the reinforcing materials. The shear-induced orientation of fillers having anisotropic geometry tends to reduce the number of interconnections between the filler particles and the degree of percolation as a consequence. Therefore, the shift in the percolation threshold at high frequencies can be attributed to the collapse of the partially interconnected CNC network.

The rheological percolation threshold can be determined by analyzing the phase homogeneity, which can be evaluated quantitatively from the slope of the Cole-Cole plot and the yield stress $\left(\tau_{y}\right)$ obtained from the Casson plot (Figure 6(c)). To be precise, a high $\tau_{y}$ and a low slope of the Cole-Cole plot indicate a high presence of the solid-like network that causes heterogeneity. ${ }^{35,36}$ Interestingly, the percolation thresholds determined from these two parameters differed from each other (Figure 6(c)). The ColeCole slopes exhibit a sudden drop from 1.2 (neat PBS) to 0.5 (CNC0.6) and level off thereafter. However, $\tau_{y}$ is drastically increased above $1.5 \mathrm{wt} \%$ CNC loading. Therefore, it can be concluded that two rheological percolation thresholds are observed at 0.6 and 1.5 wt $\%$ of CNC.

The above results reveal that the critical CNC loadings at which drastic changes occur in the mechanical and hydrolytic properties coincide with the rheological percolation thresholds. Figure 7(a) shows the phase diagram correlating the mechanical, hydrolytic and rheological properties of the PBS-CNC nanocomposites obtained by plotting $\tau_{y}$ and UTS as a function of the reinforcing efficiency of CNC. The phase diagram can be divided into three distinct zones: (1) $1^{\text {st }}$ zone: CNC loading of $0-0.8 \%$, featuring a high reinforcing efficiency, increase in the UTS, negligible increase in $\tau_{y}$, and slow chemical hydrolysis; (2) $2^{\text {nd }}$ zone: CNC loading of $0.8-1.5 \%$, featuring a moderate reinforcing efficiency, a slight decrease in the UTS, a slight increase in $\tau_{y}$, and moderate hydrolysis; and, (3) $3^{\text {rd }}$ zone: CNC loading greater than $1.5 \%$, featuring a poor reinforcing efficiency, sharp decrease in the UTS, remarkable increase in $\tau_{y}$, and fast hydrolysis. The percolation network of the CNCs developed in each zone under static or dynamic condition is schematically illustrated in Figure 7(b).

\section{Conclusion}

In this study, we demonstrated that the mechanical and hydrolytic properties of the PBS-CNC nanocomposites can be tailored based on their rheological behavior. The rheological characterizations confirmed that PBS-CNC nanocomposites exhibited two rheological percolation thresholds at approximately 0.8 and $1.5 \mathrm{wt} \%$. Interestingly, the mechanical and hydrolytic properties of PBS-CNC nanocomposites exhibit dramatic trend changes at certain critical CNC loadings. From the perspective of mechanical performance, the UTS exhibited a 1.17-fold increases at $0.8 \mathrm{wt} \%$ CNC loading when compared to neat PBS but started decreasing above this loading. In addition, the reinforcing efficiencies of CNCs calculated from Young's modulus and UTS changed significantly above $1.5 \mathrm{wt} \% \mathrm{CNC}$ loading. Chemical hydrolysis experiments revealed that the degradation of PBS-CNC nanocomposites was accelerated above $0.8 \mathrm{wt} \%$ of CNC. This study provides a rheological basis to understand and conveniently control the properties of biodegradable nanocomposites of PBS and CNC by tuning the percolation behavior of the nano-fillers (i.e., CNC).

\section{References}

(1) S. Wong, N. Ngadi, T. A. T. Abdullah, and I. M. Inuwa, Renew. Sust. Energ. Rev., 50, 1167 (2015).

(2) R. Siddique, J. Khatib, and I. Kaur, Waste Manage., 28, 1835 (2008).

(3) I. Chakraborty and P. Maity, Sci. Total Environ., 728, 138882 (2020). 
(4) J. J. Klemeš, Y. Van Fan, R. R. Tan, and P. Jiang, Renew. Sust. Energ. Rev., 127, 109883 (2020).

(5) N. Bosq and D. Aht-Ong, Macromol. Res., 26, 13 (2018).

(6) H. Kim, H. Jeon, G. Shin, M. Lee, J. Jegal, S. Y. Hwang, D. X. Oh, J. M. Koo, Y. Eom, and J. Park, Green Chem., 23, 2293 (2021).

(7) H. Kim, M. S. Shin, H. Jeon, J. M. Koo, Y. Eom, S. Choi, G. Shin, D. X. Oh, S. Y. Hwang, and J. Park, Int. J. Biol. Macromol., 173, 128 (2021).

(8) S. Lee, M. Kim, H. Y. Song, and K. Hyun, Macromolecules, 52, 7904 (2019).

(9) V. Bertolino, G. Cavallaro, G. Lazzara, S. Milioto, and F. Parisi, New J. Chem., 42, 8384 (2018).

(10) S. Singha and M. S. Hedenqvist, Polymers, 12, 1095 (2020).

(11) J. Xie, Z. Wang, Q. Zhao, Y. Yang, J. Xu, G. I. Waterhouse, K. Zhang, S. Li, P. Jin, and G. Jin, ACS omega, 3, 1187 (2018).

(12) Y. Zare, H. Garmabi, and K. Y. Rhee, Compos. Part B: Eng., 144, 1 (2018).

(13) Y. Zheng, G. Fu, B. Wang, C. Pang, P. Hall, and N. Sharmin, J. Appl. Polym. Sci., 137, 49286 (2020).

(14) M. Zahran and A. H. Marei, Int. J. Biol. Macromol, 136, 586 (2019).

(15) C. R. Estrellan and F. Iino, Chemosphere, 80, 193 (2010).

(16) S.-A. Park, Y. Eom, H. Jeon, J. M. Koo, E. S. Lee, J. Jegal, S. Y. Hwang, D. X. Oh, and J. Park, Green Chem., 21, 5212 (2019).

(17) B. Ates, S. Koytepe, A. Ulu, C. Gurses, and V. K. Thakur, Chem. Rev., 120, 9304 (2020).

(18) L. Song, Z. Wang, M. E. Lamm, L. Yuan, and C. Tang, Macromolecules, 50, 7475 (2017).

(19) L. Wang, M. Ando, M. Kubota, S. Ishihara, Y. Hikima, M. Ohshima, T. Sekiguchi, A. Sato, and H. Yano, Compos. Part A: Appl. Sci. Manuf., 98, 166 (2017).

(20) G. Kwon, K. Lee, D. Kim, Y. Jeon, U.-J. Kim, and J. You, J. Hazard. Mater.,
398, 123100 (2020).

(21) L. T. Hao, Y. Eom, T. H. Tran, J. M. Koo, J. Jegal, S. Y. Hwang, D. X. Oh, and J. Park, Nanoscale, 12, 2393 (2020).

(22) T. Saito, R. Kuramae, J. Wohlert, L. A. Berglund, and A. Isogai, Biomacromolecules, 14, 248 (2013).

(23) H. Wu, S. Nagarajan, J. Shu, T. Zhang, L. Zhou, Y. Duan, and J. Zhang, Carbohydr. Polym., 197, 204 (2018).

(24) S. M. Son, J.-E. Lee, J. Jeon, S. I. Lim, H. T. Kwon, Y. Eom, and H. G. Chae, Macromol. Res., 29, 33 (2021).

(25) T. Kim, H. Jeon, J. Jegal, J. H. Kim, H. Yang, J. Park, D. X. Oh, and S. Y. Hwang, RSC Adv., 8, 15389 (2018).

(26) A. R. de Matos Costa, A. Crocitti, L. H. d. Hecker de Carvalho, S. C. Carroccio, P. Cerruti, and G. Santagata, Polymers, 12, 2317 (2020).

(27) J. Li and Z. Qiu, Carbohydr. Polym., 205, 211 (2019).

(28) C. Li, C. Sun, C. Wang, H. Tan, Y. Xie, and Y. Zhang, Cellulose, 27, 7489 (2020).

(29) H. Shirali, M. Rafizadeh, and F. A. Taromi, Macromol. Res., 23, 755 (2015).

(30) E. Delamarche, A. Mattlet, S. Livi, J.-F. Gérard, R. Bayard, and V. Massardier, Front. Mater. Sci., 7, 7 (2020).

(31) X. Yin, S. Li, L. Wang, G. He, and Z. Yang, Polym. Korea, 41, 163 (2017).

(32) M. Barczewski and O. Mysiukiewicz, Polym. Korea, 42, 267 (2018).

(33) D. W. Chae and B. C. Kim, Macromol. Res., 18, 772 (2010).

(34) S. Wu, J. Wu, G. Huang, and H. Li, Macromol. Res., 23, 537 (2015).

(35) G. Zhang, T. Wu, W. Lin, Y. Tan, R. Chen, Z. Huang, X. Yin, and J. Qu, Compos. Sci Technol., 145, 157 (2017).

(36) P. Porkodi, J. Abhilash, H. K. Shukla, and J. Rawat, Polym. Bull., 77, 3937 (2020).

Publisher's Note Springer Nature remains neutral with regard to jurisdictional claims in published maps and institutional affiliations. 